\title{
Pregabalin-associated stuttering and frequent blepharospasm: case report and review.
}

\author{
Lingzhi Ge ${ }^{1,2} \cdot$ Ang $\mathrm{Li}^{1,3} \cdot \mathrm{Ni}$ Wang ${ }^{1} \cdot$ Ping $\mathrm{Li}^{1,4} \cdot$ Hongyan Xin ${ }^{1,5} \cdot$ Wenfei $\mathrm{Li}^{1}$
}

Received: 15 February 2020 / Accepted: 5 June 2020 / Published online: 6 July 2020

(C) The Author(s) 2020

\begin{abstract}
Herpes zoster is an acute, painful, herpes skin disease caused by varicella-zoster virus, which may cause viral meningitis. Pregabalin has been shown to be efficacious in the treatment of pain in patients with herpes zoster. However, it has the side effects of neurotoxicity. We describe a 68 -year-old female patient with herpes zoster, and she was treated with pregabalin. The patient presented with stuttering and frequent blepharospasm after 3 days of pregabalin treatment. Pregabalin was discontinued, the symptoms of stuttering and frequent blepharospasm completely resolved without any special treatment after one week. In this case, the etiology of stuttering and frequent blepharospasm may be related to pregabalin. Clinicians should be alert to the rare symptoms associated with the use of pregabalin.
\end{abstract}

Keywords Pregabalin $\cdot$ Side effects $\cdot$ Stuttering $\cdot$ Frequent blepharospasm

\section{Introduction}

Herpes zoster (HZ) is an acute, painful, herpes skin disease caused by varicella-zoster virus, which may cause viral meningitis. An important feature of $\mathrm{HZ}$ is rash-associated localized pain [1]. The extreme pain of $\mathrm{HZ}$ often drives the patient to take analgesic drugs including the alpha-2 delta ligands (pregabalin and gabapentin). It has been shown to be efficacious in the treatment of herpetic pain in patients with HZ [2]. However, the side effects of pregabalin are noteworthy to

Lingzhi Ge, Ang Li and Ni Wang contributed equally to this work.

Wenfei Li

lwf888026@163.com

1 Department of Dermatology, the First Affiliated Hospital of Shandong First Medical University, Shandong Provincial Qianfoshan Hospital, 16766 Jing-Shi Road, Jinan 250014, China

2 Department of Dermatology, the Second Affiliated Hospital of Shandong First Medical University, Tai'an 271000, China

3 Department of Orthopaedic Surgery, Shanghai Jiao Tong University Affiliated Sixth, People's Hospital, 600 Yishan Road, Shanghai 200233, China

4 Department of Dermatology, Jimo District People's Hospital, Qingdao 266200, China

5 Department of Surgery, Shandong Chest Hospital, Jinan 250013, China recognize due to its a wide range of uses. Common side effects of pregabalin reported in the published literature include peripheral edema, unsteadiness, weight gain, liver failure, altered consciousness, heart failure and rhabdomyolysis [3, 4].

Here, we describe a case of stuttering and frequent blepharospasm after pregabalin treatment. To the best of our knowledge, only one report of the patient with stuttering associated with pregabalin has been described in PubMed [5]. Our patient has not only the symptom of stuttering, but also the special clinical characteristic of frequent blepharospasm.

\section{Reason for report}

The main reason for reporting the case study is that the symptoms of stuttering and frequent blepharospasm may be related to pregabalin. That is rare and unusual. Clinicians should be alert to the rare symptoms associated with the use of pregabalin.

\section{Case presentation}

A 68-year-old Chinese woman suffered from burning pain in the skin of left lower limb and followed the skin eruption 2 days later. The eruptions initially presented as papules and plaques of erythema in the dermatome, and the plaques 
develop blisters within several hours. The lesions continued to appear for several days and reached partially confluence in the district with severe pain. She visited our department and complained about the lesions of clustered blisters and acute pain. Doctors diagnosed her with HZ by differential diagnosis. Our patient was administered $800 \mathrm{mg}$ of aciclovir chewable tablets (Shandong Zibo Xinhua Pharmaceutical Company Limited, Shandong, People's Republic of China) five times daily for 2 weeks, $75 \mathrm{mg}$ pregabalin capsules (Pfizer Pharmaceuticals Limited, New York, USA) two times daily.

To our surprise, the patient presented to our clinic with stuttering and frequent blepharospasm 3 days later. The patient had no additional signs or symptoms such as peripheral edema, unsteadiness, dizziness. She had a history of periarthritis of shoulder in the past. She felt pain in the left shoulder for several years. The pain did not decrease at rest but increases during activity. She denied taking any other medications. Related laboratory examination was carried out. White blood cell count of $6.28 \times 10^{9} / 1$ (normal: $3.5-9.5 \times$ $10^{9} / 1$ ) with $0.55 \times 10^{9} / 1$ neutrophils (normal: $0.40-0.75 \times$ $10^{9} / 1$ ); normal urine color, 6.5 PH level (normal:4.5-8.0); normal liver enzyme levels of $15.6 \mathrm{U} / 1$ AST and $19.10 \mathrm{U} / 1$ ALT (normal: 9-50 U/1, 15-40 U/1); normal electrolytes concentration of $4.23 \mathrm{mmol} / \mathrm{l}$ potassium (normal: $3.5-5.3 / \mathrm{mmol}$ ), $142 \mathrm{mmol} / \mathrm{l}$ sodium (normal: $137-147 / \mathrm{mmol}), 102 \mathrm{mmol} / \mathrm{l}$ chlorine (normal: 99-110/mmol), $2.22 \mathrm{mmol} / 1$ calcium(normal: $2.19-2.54 / \mathrm{mmol}), 0.96 \mathrm{mmol} / \mathrm{l}$ magnesium (normal: $0.70-1.10 / \mathrm{mmol})$. Neurological examination was normal. Magnetic resonance imaging of left shoulder shows that the gap between the humerus and scapula shrinking and the capsular ligament adhesion.

Pregabalin may cause or aggravate myoclonus, ataxia and other adverse events [6-8], and that is why we think that her unusual symptoms may be related to pregabalin. Pregabalin was discontinued, but aciclovir remained. We encouraged the patient to drink more water and the symptoms of stuttering and frequent blepharospasm alleviated 4 days later and completely resolved without any special treatment after pregabalin was withdrawn for 1 week.

Due to the patient felt obvious neuralgia someday 4 weeks later, she took one capsule of $75 \mathrm{mg}$ pregabalin again by herself. Unfortunately, stuttering and frequent blepharospasm occurred again, and then, pregabalin was discontinued. The symptoms of stuttering and frequent blepharospasm completely resolved 4 days later, and the neuralgia subsided after 4 months. No residual symptoms were found at a 6month follow-up.

\section{Discussion}

Pregabalin is the (S) enantiomer of 3-(aminomethyl)-5methylhexanoic acid [9]. It binds potently to the alpha-2 delta subunit, reducing calcium influx at nerve terminals, thereby, it reduces the release of glutamate, noradrenaline, and substance $\mathrm{P}$ [10]. Now pregabalin has been proven to be effective in the treatment of the neuropathic pain, just as in trigeminal neuralgia [11], and diabetic peripheral neuropathy [12].

Acute $\mathrm{HZ}$ and its complication postherpetic neuralgia (PHN) represent a significant challenge to the physicians, $11.9-14.3 \%$ of patients will develop PHN following an attack of HZ [13]. Pregabalin was approved by FDA in the treatment of HZ neuralgia in December 2004. Randomized controlled trials have shown that pregabalin not only attenuates preoperative anxiety and pain symptoms, but also improves sleep quality [14]. Given its efficacy in treating pain, pregabalin has been used more widely in patients with $\mathrm{HZ}$ neuralgia. But some side effects are gradually recognized [4].

Stuttering is a rare adverse reaction and to the authors' knowledge, only one previous case was reported in PubMed by Giray et al. in 2016 [5]. In their report, a 31-year-old female patient was assigned a diagnosis of complex regional pain syndrome. After taking of $75 \mathrm{mg}$ pregabalin twice on the first day of treatment, her speech became stuttering. The patient had no additional signs or symptoms except stuttering. She had no comorbid medical illness. One week after discontinuing pregabalin, the stuttering resolved completely. There are some differences or similarities between our patient and the patient reported. They were otherwise healthy, without abnormal laboratory tests, and the same frequency of routine administration. However, our patient presented as not only stuttering but also frequent blepharospasm, and the mechanism of frequent blepharospasm caused by pregabalin is still unknown. And due to the lack of further research, we were unable to determine whether stuttering and frequent blepharospasm was dose dependent.

People usually blink 15-20 times per minute under normal conditions, but frequent blepharospasm is a pathological phenomenon. The ophthalmological and neurological consultation revealed that our patient had no abnormalities except for blepharospasm. Our patient showed bilateral and symmetric blepharospasm that affected her daily activities. After benign essential blepharospasm was ruled out (No ophthalmologic disorders, mental stress, fatigue and others), she was diagnosed with blepharospasm associated with therapy.

Although her stuttering and frequent blepharospasm were associated with the treatment of pregabalin and aciclovir, the symptoms of stuttering and frequent blepharospasm completely resolved without any special treatment after pregabalin was withdrawn for 1 week. Therefore, we believe that pregabalin was probably responsible for her stuttering and frequent blepharospasm. Besides, we use the Naranjo scale to determine whether the adverse drug reaction is associated with the drug use rather than other factors. The drug scored 8 points on the Naranjo scale indicating that the symptom was a "probable" side effect (Table 1). 
Table 1 The correlation between adverse drug reactions and drug use measured by the Naranjo scale
Naranjo scale for our case

$\begin{array}{ll}\text { Axis } & \text { Numerical score } \\ \text { Previous reports on the reaction } & 1 \\ \text { Temporal illegibility in the onset of the reaction } & 2 \\ \text { Improvement after drug withdrawal } & 1 \\ \text { Positive re-challenge } & 2 \\ \text { Exclusion of alternative causes for the ADR } & 2 \\ \text { Placebo response } & 0 \\ \text { Drug concentration and monitoring } & 0 \\ \text { Dose relationship } & 0 \\ \text { Previous exposure and cross reactivity } & 0 \\ \text { Presence of any objective evidence } & 0 \\ \text { Total score } & 8 \\ \text { Results: }>\text { or }=9 \text { definitive; } 5-8 \text { probable; } 1-4 \text { possible; }<\text { or }=0 \text { unlikely } & \end{array}$

Studies show that the occurrence of stuttering is related to the left medial premotor cortex and dopaminergic hyperactivity within CNS $[5,15]$. It is possible that pregabalin-related stuttering might cause the imbalance between excitatory and inhibitory neurotransmission leading to dysfunction in white matter fiber tracts. Blepharospasm qualifies as a type of focal dystonia, and the site of pathology in blepharospasm remains unknown. It is possible pathogenesis that increased nigrocollicular pathway activity, and an impairment in corticosensory processing, which possibly lead to a defect of the sensorimotor gating mechanism reduced inhibition of spontaneous blinking $[16,17]$.

\section{Outcome}

The symptoms of stuttering and frequent blepharospasm completely resolved without any special treatment after pregabalin was discontinued for 1 week.

\section{Conclusion}

The case of stuttering and frequent blepharospasm may be related to pregabalin. We believe such a case is unique because no case of pregabalin-induced frequent blepharospasm has been reported. Clinicians should recognize the rare symptoms.

Acknowledgments We would like to thank Dr. Furen Zhang in Shandong Provincial Hospital for Skin Diseases and for their help.

Authors' contributions LZG, AL and NW collected, analyzed data and wrote the paper; PL and HYX collected, analyzed data; WFL designed the study, performed the data evaluation. All authors read and approved the final manuscript
Funding information This case report does not have any funding to support.

\section{Compliance with ethical standards}

Conflict of interest The authors declare that they have no competing interests.

Consent for publication Written informed consent was obtained from the patient for publication of this case report. A copy of the written consent is available for review by the Editor of this journal. This study was approved by the First Affiliated Hospital of Shandong First Medical University, Shandong Provincial Qianfoshan Hospital (QY201802016).

ICMJE form for disclosure of potential conflicts of interest This study was approved by the First Affiliated Hospital of Shandong First Medical University, Shandong Provincial Qianfoshan Hospital (QY201802016), and written informed consent has been provided by the patient to have the case details published. Conflict of interest the authors do not report any Conflict of Interest.

Abbreviations HZ Herpes zoster; PHN Postherpetic neuralgia.

Open Access This article is licensed under a Creative Commons Attribution 4.0 International License, which permits use, sharing, adaptation, distribution and reproduction in any medium or format, as long as you give appropriate credit to the original author(s) and the source, provide a link to the Creative Commons licence, and indicate if changes were made. The images or other third party material in this article are included in the article's Creative Commons licence, unless indicated otherwise in a credit line to the material. If material is not included in the article's Creative Commons licence and your intended use is not permitted by statutory regulation or exceeds the permitted use, you will need to obtain permission directly from the copyright holder. To view a copy of this licence, visit http://creativecommons.org/licenses/by/4.0/.

\section{References}

1. Parruti G, Tontodonati M, Rebuzzi C, Polilli E, Sozio F, Consorte $\mathrm{A}$, et al. Predictors of pain intensity and persistence in a prospective 
Italian cohort of patients with herpes zoster: relevance of smoking, trauma and antiviral therapy. BMC Med. 2010;8:58.

2. Parsons B, Pan X, Xie L, Chen Y, Ortiz M, Whalen E. Comparison of the efficacy and safety of pregabalin for postherpetic neuralgia in Chinese and international patients. J Pain Res. 2018;11:1699-708.

3. Kanbayashi Y, Onishi K, Hosokawa T. Factors predicting adverse events associated with pregabalin administered for neuropathic pain relief. Pain Res Manag. 2014;19(6):e164-7.

4. Kose E. Adverse drug event profile associated with pregabalin among patients with and without cancer: analysis of a spontaneous reporting database. J Clin Pharm Ther. 2018 Aug;43(4):543-9.

5. Giray E, Sanal Toprak C, Sacaklidir R, Gündüz OH. Pregabalinassociated stuttering in a patient with complex regional pain syndrome: a case report. J Clin Psychopharmacol. 2016;36(6):740-2.

6. Hellwig S, Amtage F. Pregabalin-induced cortical negative myoclonus in a patient with neuropathic pain. Epilepsy Behav. 2008;13(2):418-20.

7. Kälviäinen R, Khyuppenen J, Koskenkorva P, Eriksson K, Vanninen R, Mervaala E. Clinical picture of EPM1-UnverrichtLundborg disease. Epilepsia. 2008;49(4):549-56.

8. Oaklander AL, Buchbinder BR. Pregabalin-withdrawal encephalopathy and splenial edema: a link to high-altitude illness? Ann Neurol. 2005;58(2):309-12.

9. Hitchcock ML, Marginean I. Enantiomeric Identification of Pregabalin by GC-MS via Methylation and S-TPC Chiral Derivatization. J Forensic Sci. 2019;64(2):406-12.

10. Sabatowski R, Gãlvez R, Cherry DA, Jacquot F, Vincent E, Maisonobe $\mathrm{P}$, et al. Pregabalin reduces pain and improves sleep and mood disturbances in patients with post-herpetic neuralgia: results of a randomised, placebo-controlled clinical trial. Pain. 2004;109(1-2):26-35.
11. Hamasaki T, Yano S, Nakamura K, Yamada K. Pregabalin as a salvage preoperative treatment for refractory trigeminal neuralgia. J Clin Neurosci. 2018;47:240-4.

12. Parsons B, Li C, Emir B, Vinik AI. The efficacy of pregabalin for treating pain associated with diabetic peripheral neuropathy in subjects with type 1 or type 2 diabetes mellitus. Curr Med Res Opin. 2018;34(11):2015-22.

13. Schmidt-Ott R, Schutter U, Simon J, Nautrup BP, von Krempelhuber A, Gopala K, et al. Incidence and costs of herpes zoster and postherpetic neuralgia in German adults aged $\geq 50$ years: a prospective study. J Inf Secur. 2018;76(5):475-82.

14. Shimony N, Amit U, Minz B, Grossman R, Dany MA, Gonen L, et al. Perioperative pregabalin for reducing pain, analgesic consumption, and anxiety and enhancing sleep quality in elective neurosurgical patients: a prospective, randomized, double-blind, and controlled clinical study. J Neurosurg. 2016;125(6):1513-22.

15. Garnett EO, Chow HM, Nieto-Castañón A, Tourville JA, Guenther FH, Chang SE. Anomalous morphology in left hemisphere motor and premotor cortex of children who stutter. Brain. 2018;141(9): 2670-84.

16. Hamedani AG, Gold DR. Eyelid dysfunction in neurodegenerative, Neurogenetic, and Neurometabolic disease. Front Neurol. 2017;8: 329.

17. Fayers T, Shaw SR, Hau SC, Ezra DG. Changes in corneal aesthesiometry and the sub-basal nerve plexus in benign essential blepharospasm. Br J Ophthalmol. 2015;99(11):1509-13.

Publisher's note Springer Nature remains neutral with regard to jurisdictional claims in published maps and institutional affiliations. 